\title{
Markov properties in presence of measurement noise
}

\author{
David Kleinhans and Rudolf Friedrich \\ Westfälische Wilhelms-Universität Münster, D-48149 Münster, Germany \\ Matthias Wächter and Joachim Peinke \\ ForWind Center for Wind Energy Research, Carl-von-Ossietzky University of Oldenburg, D-26111 Oldenburg, Germany
}

(Dated: November 4, 2018)

\begin{abstract}
Recently, several powerful tools for the reconstruction of stochastic differential equations from measured data sets have been proposed [e.g. Siegert et al., Physics Letters A 243, 275 (1998); Hurn et al., Journal of Time Series Analysis 24, 45 (2003)]. Efficient application of the methods, however, generally requires Markov properties to be fulfilled. This constraint typically seems to be violated on small scales, which frequently is attributed to physical effects. On the other hand, measurement noise such as uncorrelated measurement and discretization errors has large impacts on the statistics of measurements on small scales. We demonstrate, that the presence of measurement noise, likewise, spoils Markov properties of an underlying Markov process. This fact is promising for the further development of techniques for the reconstruction of stochastic processes from measured data, since limitations at small scales might stem from artificial noise sources rather than from intrinsic properties of the dynamics of the underlying process. Measurement noise, however, can be controlled much better than the intrinsic dynamics of the underlying process.
\end{abstract}

PACS numbers: 02.50.Ga, 05.45.Tp

\section{INTRODUCTION}

Physical systems often are described by means of dynamical systems defined by differential equations of first order in time. The knowledge of a single point in phase space is sufficient for precise prediction of the future evolution of the system. Starting from this initial condition, the equations of motion can be integrated - at least numerically. Some systems are very sensitive to the initial condition and therefore are associated with deterministic chaos.

For complex systems, a deterministic description often is not feasible due to the huge amount of degrees of freedom and their frequently unknown microscopic interactions. However, in many cases the individual processes act on two different time scales. The dynamics of the entire system then can be reduced to the dynamics of some macroscopic order parameters, that enslave the highly fluctuating microscopic degrees of freedom [1]. In turn, the set of order parameters, $\boldsymbol{x}$, obeys stochastic differential equations (SDEs). If the SDEs are of first order in time, trajectories likewise can be generated from one single initial state. The evolution then does not depend on properties of the trajectory prior to the initial point and, therefore, exhibits only a very restricted memory. Realisations of particular trajectories sensitively depend on the fluctuating random forces, that are involved. However, considering an ensemble of realizations of the stochastic process, the Markovian property becomes evident.

In recent years, the analysis of stochastic time series has made great advances. Especially, the non-parametric reconstruction of the governing stochastic differential equation by means of the direct evaluation so drift and diffusion function has become a successful tool for ana- lyzing stochastic processes. A method, that initially was proposed by Siegert et al. 2], in the meantime has been applied to several problems in the field of finance [3], life sciences [4, 5, 6, 6, 8, 9, 10] and turbulence [11]. Moreover, algorithms for the efficient application of maximum likelihood methods have been developed [12, 13]. A brief overview over the estimation power of several methods can be found in 14]. Quite recently, an algorithm has been proposed, that combines the capabilities of the latter methods [15, 16]. However, the validity of Markov properties remains a crucial constraint for the efficient application of all these procedures on stationary time series data.

A close inspection of data sets generally indicates, that Markov properties are violated at small time differences. Typically, physical arguments are accounted for this effect, based on the fact that stochastic forces actually are correlated in time on small time differences. The aim of the present note is to study the influence of measurement noise on the Markov properties of measured data. We shall show that measurement noise as well interferes with and spoils the Markov properties.

The paper is organized as follows. In the next section, some methods for verification of the Markov properties of measured data sets are reconsidered. Section III contains the basic arguments concerning the influence of measurement noise on the transition probability density functions. Consequences of the central equation (6) for the Markov properties will be made explicit by means of three limiting cases, that are discussed at the end of the section. In section IV] the general results of the former section are exemplified by means of two particular examples. In detail, the impact of discretization noise on a purely deterministic system and the effects of uncorrelated measurement noise on a stochastic pro- 
cess are investigated. We conclude with section $\mathrm{V}$, which summarises the main results of our investigations and comprises the consequences for standard tools for data analysis.

\section{VERIFICATION OF MARKOV PROPERTIES}

Multivariate joint probability density functions (PDFs) are of great importance for the analysis of measured time series $\boldsymbol{x}(t)$. In principle, they contain all information on the initial data set such as spatial and temporal evolution. The benefit from a probabilistic approach on the basis of high dimensional joint PDFs, however, generally is limited.

The analysis substantially can be simplified, if the data set under consideration satisfies Markov properties. This circumstance is equivalent to the representation of all multivariate joint PDFs in products of single-conditioned PDFs,

$$
\begin{gathered}
P_{n}\left(\boldsymbol{x}_{\boldsymbol{n}}, \boldsymbol{x}_{\boldsymbol{n}-\mathbf{1}}, \ldots, \boldsymbol{x}_{\mathbf{0}}\right)=P_{2}\left(\boldsymbol{x}_{\boldsymbol{n}} \mid \boldsymbol{x}_{\boldsymbol{n}-\mathbf{1}}\right) \\
\times \ldots \times P_{2}\left(\boldsymbol{x}_{\mathbf{1}} \mid \boldsymbol{x}_{\mathbf{0}}\right) P_{1}\left(\boldsymbol{x}_{\mathbf{0}}\right) .
\end{gathered}
$$

Here, $P_{1}\left(\boldsymbol{x}_{\boldsymbol{i}}\right)$ is a shorthand notation for the probability of being at time $t_{i}$ in a small interval at $\boldsymbol{x}_{\boldsymbol{i}}$ with $t_{i}<t_{i+1} \forall i$. In general, the latter transition PDFs furthermore explicitly depend on the times $t_{n}, \ldots, t_{0}$.

Let us now assume the sample to be ergodic and stationary in a sense, that ensemble averages can be carried out by means of time averages and the PDFs do not depend on time explicitly [17]. Then, the ChapmanKolmogorov equation [17, 18]

$$
P\left(\boldsymbol{x}_{\boldsymbol{i}} \mid \boldsymbol{x}_{\boldsymbol{i}-\mathbf{2}}\right)=\int d \boldsymbol{x}_{\boldsymbol{i - 1}} P\left(\boldsymbol{x}_{\boldsymbol{i}} \mid \boldsymbol{x}_{\boldsymbol{i}-\mathbf{1}}\right) P\left(\boldsymbol{x}_{\boldsymbol{i - 1}} \mid \boldsymbol{x}_{\boldsymbol{i - 2}}\right),
$$

has to be fulfilled for any Markov process. This equation can be evaluated numerically for measured data sets. Although the validation of this equation is not sufficient for the validity of Markov properties, it has turned out to be a very robust criterion.

Moreover, a direct comparison of the conditional probability distributions $P\left(\mathbf{x}_{2} \mid \mathbf{x}_{1}, \mathbf{x}_{0}\right)$ and $P\left(\mathbf{x}_{2} \mid \mathbf{x}_{1}\right)$ has been used for validation of Markov properties. For Markovian data, these functions should coincidence for arbitrary values of $\mathbf{x}_{0}$. An example for the application of this procedure by means of graphical inspection of the PDFs is depicted in figure 1, that has been prepared by Wächter et al. in connection with the study of the statistical properties of hight profiles of gold surfaces [10]. Here, Wächter et al. investigated Markov properties of the transition PDFs for nested heigth increments in different scales. In the present case, Markov properties might be fulfilled for scales separated by $\Delta r=35 \mathrm{~nm}$, whereas they evidently are violated at separation lenghts of $\Delta r=14 \mathrm{~nm}$, as can be seen from inspection of figure 1. It is evident, that the proper interpretation of the plot with respect to the Markov properties has to be quantified by introducing a certain measure for the distance of the two probability distributions. To this end the Wilcoxon test [19, 20] can be applied in order to compare PDFs, that originate from samples of different size, and only makes few demands on the properties of the individual PDFs. The numerical implementation is straightforward, results for the present example e.g. are depicted in [10]. For a detailed description of the Wilcoxon test we refer to the appendix of 21].

If the direct estimation [2] of drift and diffusion functions from measured data sets is intended and the underlying process, therefore, is assumed to obey Langevin equations, an alternative method can be applied for inspection of Markov properties. Once the estimation procedure has been performed and an estimate for drift and diffusion functions is available, the character of the dynamical noise can be determined from the sample. The presence of noise without any temporal and spatial correlations is a sufficient indication for compliance of the measured data set with Markov properties. This procedure e.g. is outlined and applied in 22]. It is certainly the most direct way to investigate Markov properties.

\section{IMPACT OF MEASUREMENT NOISE ON MARKOV PROPERTIES}

An ensemble of Markov processes $x(t)$ is considered, that now is distorted by measurement noise $\xi(t)$. For simplicity, the details are carried out for a one-dimensional process. Only three consecutive points $x_{0}, x_{1}$ and $x_{2}$ with $x_{i}:=x\left(t_{i}\right)$ and $t_{i}:=t_{0}+i \tau$ are investigated for this purpose. Since the statistics is assumed to be stationary, this is sufficient for the current considerations. Henceforth, $P_{x}\left(x_{i+1} \mid x_{i}\right)$ is a shorthand notation for the transition PDF of the variable $x$ in the time increment $\tau$.

Let us now assume, that the true process is hidden to the data analyst: Instead of the variable $x(t)$, a perturbed variable $y(t)$ is measured, that emerges from the initial process by means of the relation

$$
y(t)=x(t)+\xi(x(t), t) \quad .
$$

Thereby, $\xi(x(t), t)$ is a stochastic variable, that incorporates systematic and non-systematic measurement errors. We further assume, that the deterministic contributions to the measurement error can be identified and the noise $\xi$ can be specified by

$$
\xi(x(t), t)=\xi_{s}\left(x(t)+\xi_{n s}(t)\right) .
$$

Here, $\xi_{n s}$ incorporates non-systematic noise sources. For reasons of simplicity, we assume these errors to be independent of one another for consecutive measurements,

$$
\left\langle\xi_{n s}(t+\tau) \xi_{n s}(t)\right\rangle \sim \delta(\tau) \quad .
$$

On the other hand, $\xi_{s}$ characterises deterministic, systematic measurements errors, that have no explicit de- 

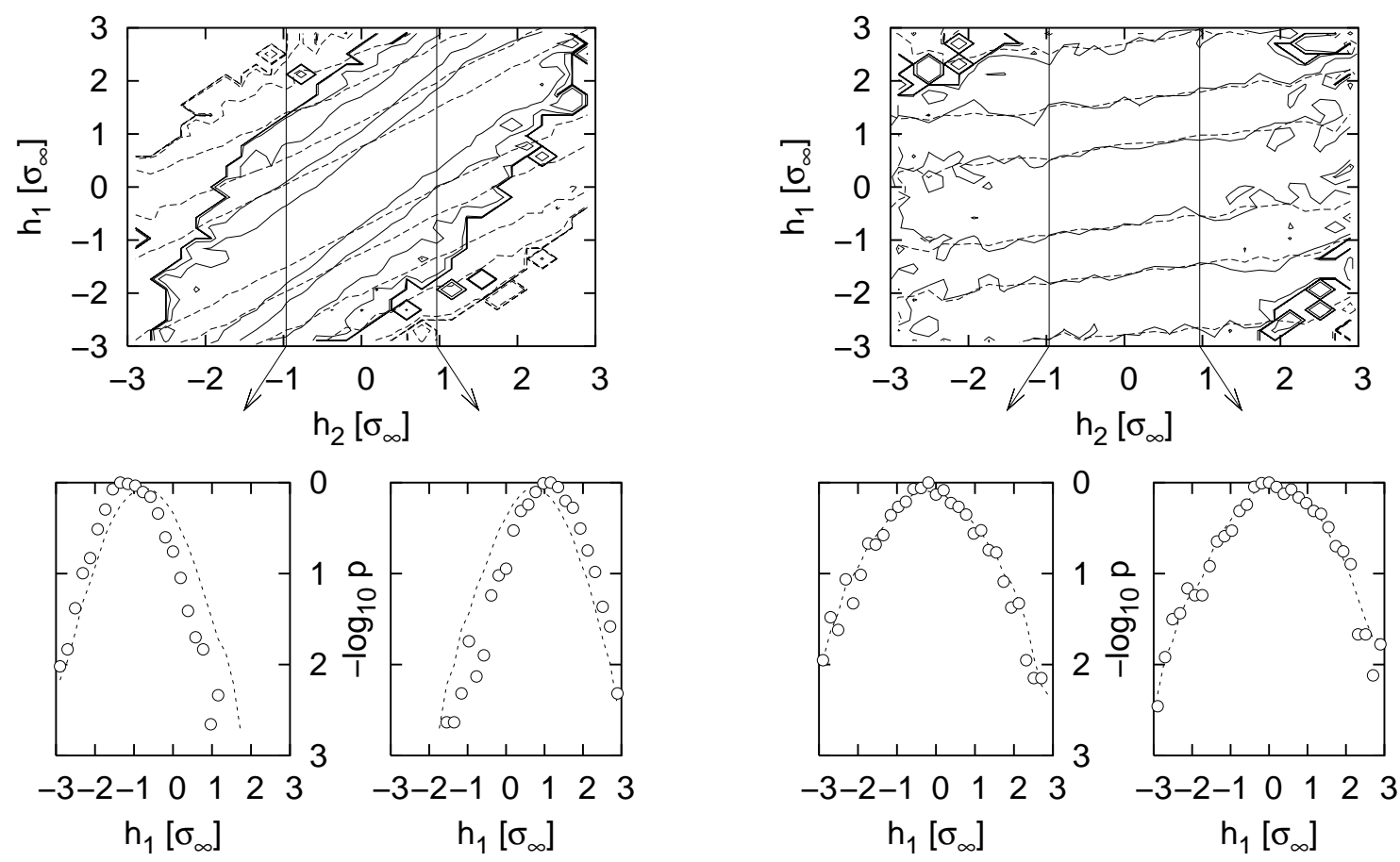

FIG. 1: Example for the analysis of Markov properties by means of graphical inspection of transition PDFs, prepared by Wächter et al. [10]. Test for Markov properties of Au film data for two different scale separations $\Delta r=14 \mathrm{~nm}$ (lhs) and $35 \mathrm{~nm}$ (rhs), where $\Delta r=r_{3}-r_{2}=r_{2}-r_{1}$. In both cases $r_{2}=169 \mathrm{~nm}$. In each case a contour plot of conditional probabilities $P\left(h_{1}, r_{1} \mid h_{2}, r_{2}\right)$ (dashed lines) and $P\left(h_{1}, r_{1} \mid h_{2}, r_{2} ; h_{3}=0, r_{3}\right)$ (solid lines) is shown in the top panel. Contour levels differ by a factor of 10, with an additional level at $p=0.3$. Below the top panels in each case, two one-dimensional cuts at $h_{2} \approx \pm \sigma_{\infty}$ are shown with $P\left(h_{1}, r_{1} \mid h_{2}, r_{2}\right)$ as dashed lines and $P\left(h_{1}, r_{1} \mid h_{2}, r_{2} ; h_{3}=0, r_{3}\right)$ as circles. From the deviations of the PDFs for $\Delta r=14 \mathrm{~nm}$ (lhs) it becomes evident, that Markov properties are not fulfilled in this case. They might, however, be valid for $\Delta r=35 \mathrm{~nm}$ (rhs).

pendence on $t$. We would like to emphasize, that discretization errors fall into this broad class, that are an intrinsic feature of any digital measurement procedure. While the former noise is uncorrelated, this assumption generally is violated for the latter noise source due to correlations in the variable $x$ itself.

The probability for the measurement of $y_{i}$ now solely depends on the entangled variable $x_{i}$ and can be specified by means of the conditional probability $P_{\xi}\left(y_{i} \mid x_{i}\right)$. Hence, the conditional probability $P_{y}\left(y_{2} \mid y_{1}, y_{0}\right)$ for the process $y(t)$ can be calculated by means of its definition through joint probabilities. Application of the Markov properties of the underlying process $x(t)$ finally yields

$$
P_{y}\left(y_{2} \mid y_{1}, y_{0}\right)=\frac{P_{y}\left(y_{2}, y_{1}, y_{0}\right)}{P_{y}\left(y_{1}, y_{0}\right)}=\frac{\int d x_{2} \int d x_{1} \int d x_{0} P_{\xi}\left(y_{2} \mid x_{2}\right) P_{\xi}\left(y_{1} \mid x_{1}\right) P_{\xi}\left(y_{0} \mid x_{0}\right) P_{x}\left(x_{2} \mid x_{1}\right) P_{x}\left(x_{1} \mid x_{0}\right) P_{x}\left(x_{0}\right)}{\int d x_{1} \int d x_{0} P_{\xi}\left(y_{1} \mid x_{1}\right) P_{\xi}\left(y_{0} \mid x_{0}\right) P_{x}\left(x_{1} \mid x_{0}\right) P_{x}\left(x_{0}\right)}
$$

In general, this expression deviates from the single conditioned PDF $P_{y}\left(y_{2} \mid y_{1}\right)$. Therefore, noisy measurements on perfect Markov processes in general lose their Markov property due to the inexact measurement procedure.

Referring to section \ this means, that a single point from a noisy measurement on a Markov process, $y\left(t_{n}\right)$, not in any case is sufficient for a proper prediction of the future dynamics of the measured data. This makes sense, since the intrinsic state of the system, $x\left(t_{n}\right)$, hardly can be estimated from just one single measurement due to the measurement uncertainty. Rather, the consideration of a couple of noisy measurements, $y\left(t_{0}\right), \ldots, y\left(t_{n}\right)$, can enhance the accuracy of the predicted probability of $y\left(t_{n+1}\right)$. 
At least for three simple cases, expression (6) can be investigated analytically.

First, Markov properties are retrieved for the trivial case $P_{\xi}(y \mid x)=\delta(y-x)$, where actually no measurement noise is present.

Second, (6) can be evaluated for $P_{x}\left(x_{i+1} \mid x_{i}\right)=$ $P_{x}\left(x_{i+1}\right)$. In this case, the entangled process itself does not show any correlations. Frequently, this approximately is true for large time increments between individual measurements. If so, the integrals disentangle and the noisy measurements themselves turn out to be independent of one another, $P_{y}\left(y_{2} \mid y_{1}, y_{0}\right)=P_{y}\left(y_{2}\right)$. Thus, the measured variable $y$ satisfies Markov properties.

Third, noisy measurements can be considered, that sample the process much faster than the intrinsic dynamics of the entangled variable, $x$. Therefore, $P_{x}\left(x_{2} \mid x_{1}\right)=$ $\delta\left(x_{2}-x_{1}\right)$ is a reasonable approximation of the transition PDF on consecutive measurements. Moreover, only purely non-systematic, Gaussian measurement noise with variance $\sigma^{2}$ is taken into account. In this case, evaluation of expression (6) yields

$$
\begin{gathered}
P_{y}\left(y_{2} \mid y_{1}, y_{0}\right)=\sqrt{\frac{1}{3 \pi \sigma^{2}}} \exp \left[-\frac{\left(2 y_{2}-y_{1}-y_{0}\right)^{2}}{12 \sigma^{2}}\right] \\
\times \frac{\int d x_{0} \sqrt{\frac{1}{2 \pi \sigma^{2} / 3}} \exp \left[-\frac{\left(x_{0}-\frac{1}{3}\left(y_{2}+y_{1}+y_{0}\right)\right)^{2}}{2 \sigma^{2} / 3}\right] P_{x}\left(x_{0}\right)}{\int d x_{0} \sqrt{\frac{1}{2 \pi \sigma^{2} / 2}} \exp \left[-\frac{\left(x_{0}-\frac{1}{2}\left(y_{1}+y_{0}\right)\right)^{2}}{2 \sigma^{2} / 2}\right] P_{x}\left(x_{0}\right)} .
\end{gathered}
$$

In the latter factor, two different convolutions occur in numerator and denominator: The stationary PDF $P_{x}\left(x_{0}\right)$ is convoluted with Gaussian PDFs with different standard deviations, centred at the average value of $y_{2}, y_{1}, y_{0}$ and $y_{1}, y_{0}$, respectively. Therefore, this expression generally depends on the value $y_{0}$ and conflicts with Markov properties of $y(t)$. We would like to emphasize, that the approximation of a persistent entangled process is feasible for fast but noisy measurements on rather slow processes. The current case reveals the loss of Markov properties on the very small time scales for these kind of measurements, that does not stem from its intrinsic dynamics but, purely, from uncertainties during the measurement process.

\section{EXAMPLES}

Let us now elucidate the findings of the latter section by means of two examples. First, the influence of discretization noise on the properties of a simple deterministic process is investigated. By construction, the violation of the Chapman-Kolmogorov equation can be demonstrated.

Second, the influence of Gaussian measurement noise on the Markov properties of a stochastic process at relatively high time lag is considered. The effect of measurement noise becomes obvious from the inspection of conditional PDFs obtained by numerical integration of the Chapman-Kolmogorov equation (2).

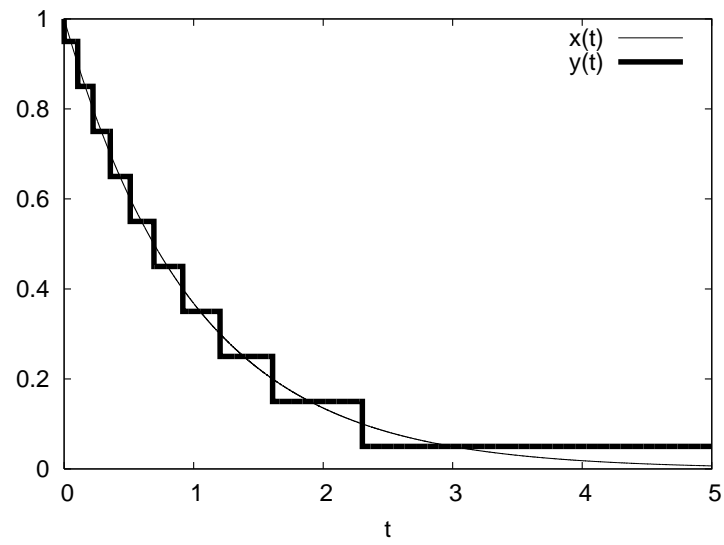

FIG. 2: Example of a process $x(t)$ according to eqn. (8), that is affected by strong discretization noise. Here, the faint line specifies the original process $x(t)$, whereas the bold line depicts the evolution $y(t)$, that eventually is obtained from the measurement due to discretization errors.

\section{A. Influence of discretization noise on a deterministic process}

We consider the elementary process

$$
x\left(t_{0}+\tau\right)=x\left(t_{0}\right) \exp [-\gamma \tau] .
$$

It is the general solution of the ordinary differential equation $\dot{x}=-\gamma x$. Since the dynamics are of first order in time, the one dimensional process $x(t)$ can be specified by one initial condition and, therefore, is Markovian. Due to the deterministic character, the conditional transition PDF for the variable $x$ in the time interval $\tau$ complies with

$$
P_{x}\left(x_{1} \mid x_{0}, \tau\right)=\delta\left(x_{1}-x_{0} e^{-\gamma \tau}\right) \quad .
$$

The process apparently is not stationary, since no forcing is present. The conditional transition PDFs, however, do not depend on time explicitly. We now consider the statistics of an ensemble of measurements, whose initial positions $x\left(t_{0}\right)$ of the individual processes are distributed according to $P_{x}(x)$.

We assume that the exact intrinsic variable $x$ is entangled due to discretization errors, that occur during an imaginary measurement procedure. Therefore, the exact, continuous variables $x$ are mapped to a finite set of discrete variables $\Omega=\left\{\omega_{0}, \ldots, \omega_{n}\right\}$ according to the rule

$$
x \rightarrow y=\omega_{i} \text { such that } \omega_{i}^{-} \leq x<\omega_{i}^{+} .
$$

Here, the intervals $\left[\omega_{i}^{-}, \omega_{i}^{+}\right]$and $\left[\omega_{i+1}^{-}, \omega_{i+1}^{+}\right]$associated with the variables $\omega_{i}$ and $\omega_{i+1}$ are connected to one another by the requirements $\omega_{i}^{+}=\omega_{i+1}^{-}$and $\omega_{i}^{-}<\omega_{i}^{+}$. Moreover it is implied, that any measured value $x$ can be mapped by means of (10). The interval $\left[\omega_{0}^{-}, \omega_{n}^{+}\right]$, thus, covers all values $x(t)$ that are realised by any process under consideration at any time $t$. The discretization noise 
can be specified in compliance with the notation of the latter section by the conditional PDF

$$
P_{\xi}(y \mid x)=\left\{\begin{array}{lll}
1 & \text { if } & x \in\left[y^{-},<y^{+}[\right. \\
0 & \text { if } x \notin\left[y^{-},<y^{+}[\right.
\end{array} .\right.
$$

The effect of discretization noise on the initial variable $x$ is illustrated in figure 2 As $y$ only assumes discrete values $\omega_{0}, \ldots, \omega_{n}$, the normalisation of the latter PDF for any $x$ is guaranteed by the equation

$$
\sum_{y \in \Omega} P_{\xi}\left(y_{i} \mid x\right)=1
$$

We now would like to demonstrate the loss of Markov properties due to the discretization of the signal. In principle, eqn. (6) directly could be evaluated numerically for the ensemble under consideration. However, in this case the invalidity of the Chapman-Kolmogorov equation (2) nicely can be utilized for this purpose.

Analogous to eqn. (6), the transition PDF conditioned on a single point can be specified,

$$
\begin{aligned}
& P_{y}\left(y_{1} \mid y_{0}, \tau\right) \\
& =\frac{\int d x_{1} \int d x_{0} P_{\xi}\left(y_{1} \mid x_{1}\right) P_{\xi}\left(y_{0} \mid x_{0}\right) P_{x}\left(x_{1} \mid x_{0}, \tau\right) P_{x}\left(x_{0}\right)}{\int d x_{0} P_{\xi}\left(y_{0} \mid x_{0}, \tau\right) P_{x}\left(x_{0}\right)} .
\end{aligned}
$$

Application of the particular transition PDFs (9) and (11) yields

$$
P_{y}\left(y_{1} \mid y_{0}, \tau\right)=\frac{\int_{\max \left(y_{0}^{-}, e^{\gamma \tau} y_{1}^{-}\right)}^{\min \left(y_{0}^{+}, \gamma^{\gamma \tau} y^{+}\right)} P_{x}\left(x_{0}\right)}{\int_{y_{0}^{-}}^{y^{+}} d x_{0} P_{x}\left(x_{0}\right)} .
$$

If the process $y(t)$ would obey Markov properties, the discrete version of the Chapman-Kolmogorov equation,

$$
P_{y}\left(y_{2} \mid y_{0}, 2 \tau\right)=\sum_{y_{1} \in \Omega} P_{y}\left(y_{2} \mid y_{1}, \tau\right) P_{y}\left(y_{1} \mid y_{0}, \tau\right)
$$

would have to be fulfilled for any choice of $y_{2}, y_{0}$ and $\tau$. For $y_{2}=y_{0}=y$ with $y^{-}>0$ and $\tau=\log \left(y^{+} / y^{-}\right) /(2 \gamma)$, the invalidity of this equation is evident, if $P_{x}(x)>0$ for $x \in\left[y^{-}, y^{+}\right]$: The left hand side of eqn. (15) vanishes, whereas the sum on the right hand side involves the summand

$$
\left[\frac{\int_{e^{\log \left(y^{+} / y^{-}\right) / 2} y^{-}}^{y^{+}} d x_{0} P_{x}\left(x_{0}\right)}{\int_{y^{-}}^{y^{+}} d x_{0} P_{x}\left(x_{0}\right)}\right]^{2}>0 .
$$

As the other summands are non-negative, the ChapmanKolmogorov equation is violated for the process under consideration. Consequently, the distorted process $y(t)$ does not comply with Markov properties any more.

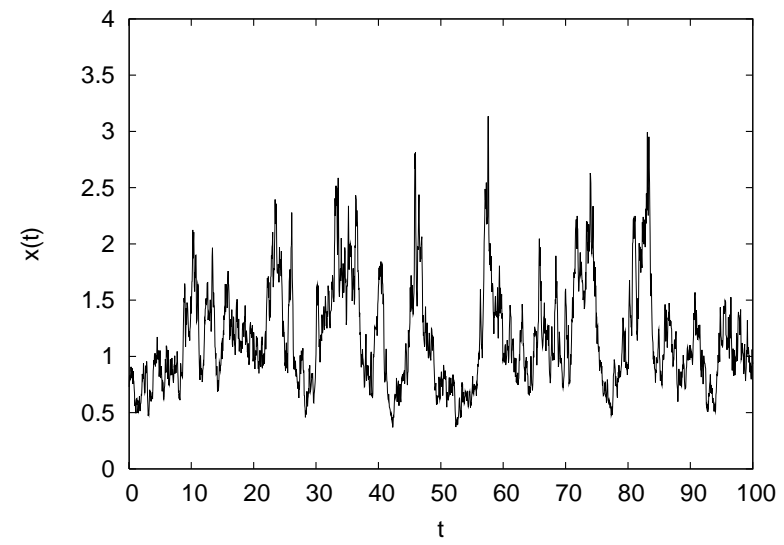

FIG. 3: Detail of a sample path of the stochastic process (17) for the parameters $(\gamma, D)=(0.75,0.1)$. The occurrence of distinct peaks is characteristic for multiplicative stochastic processes.

\section{B. Influence of measurement noise on a stochastic process}

The influence of Gaussian measurement noise on an one dimensional stochastic process with drift and diffusion functions

$$
\begin{aligned}
& D^{(1)}(x)=x\left(D-\gamma \log \left(\frac{x}{x_{0}}\right)\right) \\
& D^{(2)}(x)=D x^{2}
\end{aligned}
$$

is investigated. For further details on stochastic processes we refer to 17, 18]. This process already has been discussed in [16] within the scope of an analytical example. Thereby, the following procedure for the exact simulation of a discrete sample of this process by means of the underlying Ornstein-Uhlenbeck process $s(t)$ has been motivated,

$$
\begin{aligned}
x_{i} & =\exp \left[s_{i}\right] \\
s_{i+1} & =e^{-\gamma \Delta t} s_{i}+\sqrt{\frac{D}{\gamma}\left(1-e^{-2 \gamma \tau}\right)} \Gamma_{i} .
\end{aligned}
$$

Here, equation (18b) is the rule for the discrete simulation of an underlying Ornstein-Uhlenbeck process $s$, where $\Gamma_{i}$ are normally distributed independent random variables with variance 1 . It is deduced from the transition PDFs for the Ornstein-Uhlenbeck process, that exactly can be specified even for finite time lag $\tau$ [18]. In this vein, discretization errors stemming from the standard schemes for the numerical integration of SDEs [23] are avoided. The starting value $s_{0}$ should be drawn from a Gaussian distribution with variance $D / \gamma$, which is the stationary distribution of the process $s$. The desired process $x$ is obtained from the process $s$ by means of the nonlinear transform (18a). A sample process for parameter set $(\gamma, D)=(0.75,0.1)$ is depicted in figure 3 . 

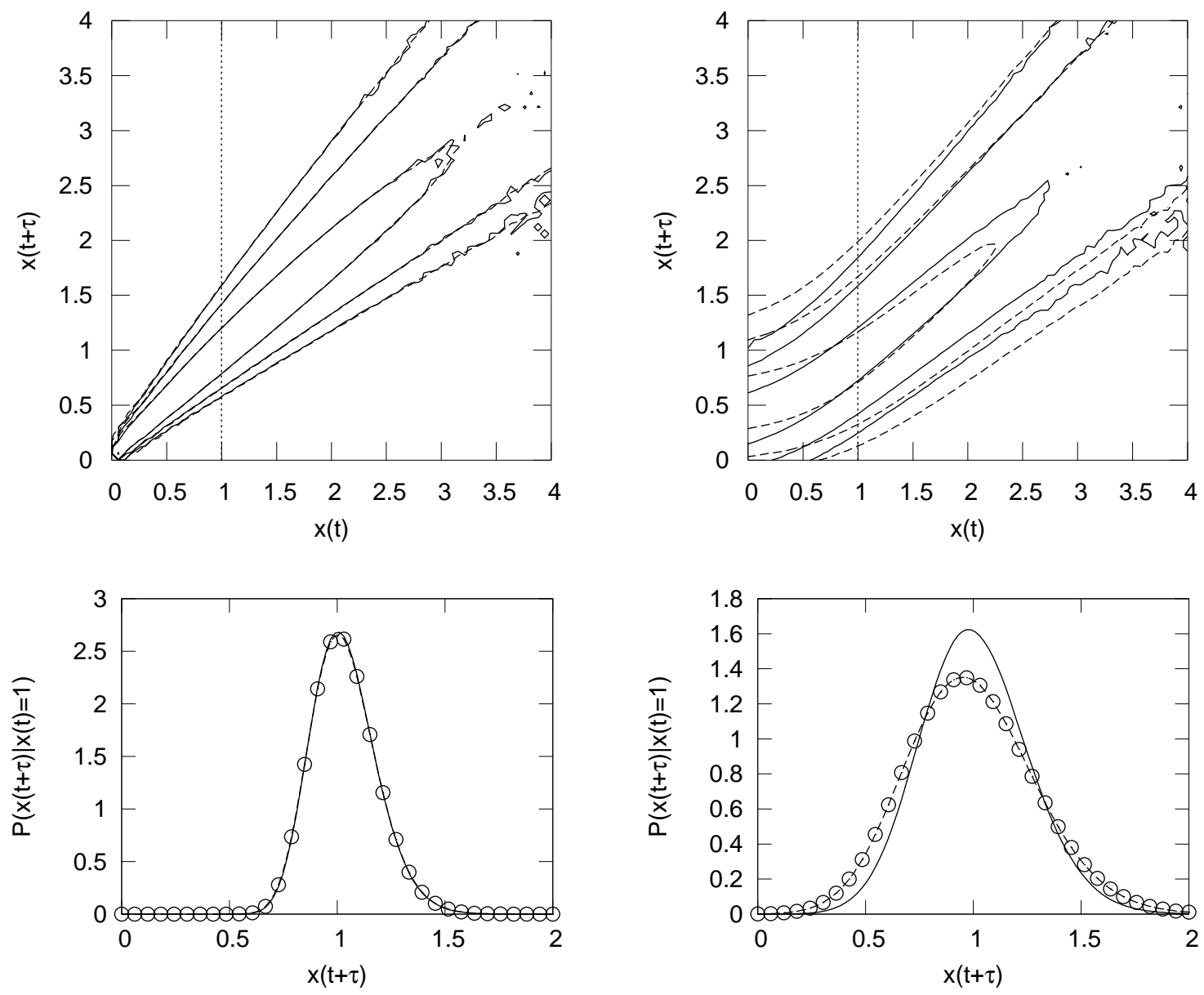

FIG. 4: Test for Markov properties for simulated samples A without measurement noise (lhs) and B with artificial Gaussian measurement noise with variance $2.25 \cdot 10^{-2}$ (rhs), respectively. In the upper panels, the conditional transition PDFs for $\tau=0.1$ (solid contour lines) are compared with the ones obtained for the same time increment by numerical integration of the Chapman-Kolmogorov equation (2) for transition PDFs for increment $\tau / 2$ (dashed contour lines). Contour lines are placed at the levels 10, 1, 0.1 and 0.01. In the lower panels, a cross section of the transition PDF at $x(t)=1$ is depicted. For reasons of clearness, circles have been added to the dashed lines corresponding to the data set B. Perfect coincidence of the PDFs is observed for A, whereas in case of B systematic deviations become evident. Consequently, Markov properties are spoiled by the artificial measurement noise of sample B.

For the current example, time series A consisting of $50 \cdot 10^{6}$ sample points with time increment $\tau=0.05$ was simulated. A second series, B, was generated from series $\mathrm{A}$ by addition of independent, identically distributed Gaussian random variables with variance $2.25 \cdot 10^{-2}$, that model noise stemming from non-systematic measurement errors. Both series A and B have been subjected to the same analyzing procedure: Conditional PDFs have been calculated from the for time increment $\tau=0.1$. On the other hand these conditional PDFs have been calculated from conditional PDFs for the time lag $\tau=0.05$ by means of numerical integration of the Chapman-Kolmogorov equation, (2). The results are exhibited in figure 4. In theory, these PDFs should coincidence with the former ones for Markovian processes. However, distinctive systematic deviations show up in presence of measurement noise, as can be seen from the analysis of data set B in figure 4. Hence, the artificial measurement noise of time series B interferes with the Markov properties of the underlying time series A.

The sets $\mathrm{A}$ and $\mathrm{B}$ correspond to the first and third limiting case of equation (6), respectively, that were discussed at the end of section [II] The second case also can be investigated by means of the current example with an increased time lag $\tau$, such that $\exp (-\gamma \tau) \ll 1$. Then, Markov properties are reobtained even in case of strong measurement noise.

\section{CONCLUSION}

The influence of different noise sources on the structure of multivariate joint probability distribution functions has been investigated. In particular, the effects of noise on the sensitivity of transition probability density functions to an additional, second condition has been 
analysed. It turned out, that noise generally has impacts on these transition probability density functions and seriously interferes with Markov properties, even if they are fulfilled for the original, uncorrupted process. This fact is, in our opinion, counter-intuitive.

The analysis of samples, that are affected by measurement noise, already for a long time is routine in applied sciences and industrial applications. Typically, Kalman filtering is applied fur this purpose [24]. For a recent review on this and other iterative techniques we refer to [25]. Recently, Siefert et al. [26] addressed this problem from a dynamical systems' point of view. The intention was to extend the efficient non-parametric estimation procedure proposed by Siegert and al. [2] to data suffering from measurement noise. In this context it could be shown, that intrinsic dynamical and external measurement noise in principle can be separated from one another, if the sampling frequency is sufficiently high whereas the amplitude of the measurement noise is weak.
Following, Böttcher et al. succeeded in the efficient reconstruction of simple processes even in presence of strong measurement noise [27]. Although the latter work is based on eqn. (13), the general problem of the vanishing Markov properties in presence of measurement noise could not be identified. This new point of view, however, involves a broad class of tools that are available for data analysis, since most tools rely on a finite embedding of the data.

The new insights have consequences for future analysis of time series: The influence of measurement noise should be discussed for any individual method, that is applied for the analysis of time series. Explicitly, also effects stemming from discretization errors should be considered here. Eventually, methods might be applicable even to data sets, that until now could not be processed due to invalidity of Markov properties. This feature, however, might stem from artificial noise rather than from intrinsic properties of the dynamics of the underlying process.
[1] H. Haken, Synergetics, Springer Series in Synergetics (Springer-Verlag, Berlin, 2004), pp. xvi+763, introduction and advanced topics, Reprint of the third (1983) edition [Synergetics] and the first (1983) edition [Advanced synergetics].

[2] S. Siegert, R. Friedrich, and J. Peinke, Physics Letters A 243, 275 (1998).

[3] R. Friedrich, J. Peinke, and C. Renner, Phys. Rev. Lett 84, 5224 (2000).

[4] S. Kriso, R. Friedrich, J. Peinke, and P. Wagner, Physics Letters A 299, 287 (2002).

[5] T. Kuusela, Physical Review E 69, 031916 (2004).

[6] P. Sura and J. Barsugli, Phys. Lett. A 305, 304 (2002).

[7] K. Ivanova, H. N. Shirer, and E. E. Clothiaux, Journal of Geophysical Research 111, (2006).

[8] J. Prusseit and K. Lehnertz, Physical Review Letters 98, 138103 (2007).

[9] G. R. Jafari et al., Physical Review Letters 91, 226101 (2003).

[10] M. Wächter et al., The European Physical Journal B 41, 259 (2004).

[11] R. Friedrich and J. Peinke, Phys. Rev. Lett. 78, 863 (1997).

[12] H. Akaike, Journal of Econometrics 16, 3 (1981).

[13] Y. Ait-Sahalia, Econometrica 70, 223 (2002).

[14] A. S. Hurn, K. A. Lindsay, and V. L. Martin, Journal of Time Series Analysis 24, 45 (2003).

[15] D. Kleinhans, R. Friedrich, A. Nawroth, and J. Peinke, Phys Lett A 346, 42 (2005).

[16] D. Kleinhans and R. Friedrich, Maximum Likelihood Es- timation of Drift and Diffusion Functions, 2006, preprint available at http://arXiv.org/abs/physics/0611102, to appear in Physics Letters A.

[17] C. W. Gardiner, Handbook of stochastic methods for physics, chemistry and the natural sciences, Vol. 13 of Springer Series in Synergetics, 3rd ed. (Springer-Verlag, Berlin, 2004), pp. xviii+415.

[18] H. Risken, The Fokker-Planck equation, Vol. 18 of Springer Series in Synergetics, 2nd ed. (Springer-Verlag, Berlin, 1989), pp. xiv +472 , methods of solution and applications.

[19] F. Wilcoxon, Biometrics Bulletin 1, 80 (1945).

[20] H. B. Mann and D. R. Whitney, The Annals of Mathematical Statistics 18, 50 (1947).

[21] C. Renner, J. Peinke, and R. Friedrich, J. Fluid Mech. 433, 383 (2001).

[22] P. Marcq and A. Naert, Physics of Fluids 13, 2590 (2001).

[23] P. E. Kloeden and E. Platen, Numerical solution of stochastic differential equations, Vol. 23 of Applications of Mathematics (New York) (Springer-Verlag, Berlin, 1992), pp. xxxvi+632.

[24] R. E. Kalman, Transactions of the ASME-Journal of Basic Engineering 82, 35 (1960).

[25] H. U. Voss, J. Timmer, and J. Kurths, International Journal of Bifurcation and Chaos 14, 1905 (2004).

[26] M. Siefert, A. Kittel, R. Friedrich, and J. Peinke, Europhys. Lett. 61, 466 (2003).

[27] F. Böttcher et al., Phys. Rev. Lett. 97, 090603 (2006). 